Revue d'archéologie préhistorique

\title{
Percussion bipolaire sur enclume : choix ou contrainte ? L'exemple du Paléolithique d'Ounjougou (Pays dogon, Mali)
}

The bipolar-on-anvil technique: choice or constraint? An example from the Palaeolithic of Ounjougou (Dogon country, Mali)

Sylvain Soriano, Aline Robert et Éric Huysecom

\section{OpenEdition \\ Journals}

Édition électronique

URL : http://journals.openedition.org/paleo/1962

DOI : $10.4000 /$ paleo. 1962

ISSN : 2101-0420

Éditeur

SAMRA

Édition imprimée

Pagination : 123-132

ISSN : 1145-3370

Référence électronique

Sylvain Soriano, Aline Robert et Éric Huysecom, «Percussion bipolaire sur enclume : choix ou contrainte? L'exemple du Paléolithique d'Ounjougou (Pays dogon, Mali) », PALEO [En ligne], Numéro spécial | 2009-2010, mis en ligne le 23 avril 2012, consulté le 07 juillet 2020. URL : http:// journals.openedition.org/paleo/1962 ; DOI : https://doi.org/10.4000/paleo.1962

\section{(c) (i) $\ominus$}

PALEO est mis à disposition selon les termes de la licence Creative Commons Attribution - Pas d'Utilisation Commerciale - Pas de Modification 4.0 International. 


\title{
PERCUSSION BIPOLAIRE SUR ENCLUME : CHOIX OU CONTRAINTE ? L'EXEMPLE DU PALÉOLITHIQUE D'OUNJOUGOU (Pays dogon, Mali)
}

\author{
Sylvain SORIANO(1), Aline ROBERT ${ }^{(2)}$ et Éric HUYSECOM ${ }^{(3)}$
}

\begin{abstract}
Résumé : Encore trop souvent considérée comme relevant d'une production lithique expédiente, la percussion bipolaire sur enclume occupe cependant une place majeure dans les industries de certaines régions. Ainsi à Ounjougou (Pays dogon, Mali), ce mode de taille est régulièrement présent au sein des industries d'une séquence chronoculturelle qui s'étale sur plus de 100000 ans, couvrant l'essentiel du Paléolithique moyen régional. Le recours à ce mode de taille pourrait être simplement explicité par la faible diversité des ressources en matières premières et par la place qu'y occupe le quartz. Cependant, la percussion bipolaire sur enclume est totalement absente dans certaines industries de la séquence et le déterminisme des matières premières n'apparaît ainsi pas seul en jeu. La profondeur diachronique de la séquence d'Ounjougou donne l'opportunité de s'interroger sur le rôle technique, le statut économique et la charge culturelle de ce mode de taille original qu'est la percussion bipolaire sur enclume.
\end{abstract}

Mots-clés : Afrique de l'Ouest, Paléolithique, technologie lithique, technique de débitage, enclume.

Abstract: The bipolar-on-anvil technique: choice or constraint? An example from the Palaeolithic of Ounjougou (Dogon country, Mali). While too often considered as reflecting expedient lithic production, the bipolar on anvil technique is, however, a major component in the industries of certain regions. At Ounjougou (Dogon Country, Mali), this reduction technique is consistently represented in the industries of a chrono-cultural sequence extending over more than 100,000 years, covering nearly the whole of the regional Middle Paleolithic. Recourse to bipolar percussion could simply be explained by the low diversity in raw materials and the role played by quartz. However, it is entirely absent in certain industries of the sequence and raw material thus does not appear to be the only factor affecting choice of reduction technique. The temporal depth of the Ounjougou sequence offers the opportunity to investigate the technological role, economic status and cultural significance of the bipolar on anvil technique.

Key-words: West Africa, Paleolithic, lithic technology, flaking technique, anvil.

II serait simple et tentant de mettre en relation l'usage, par les Paléolithiques, de techniques et méthodes de débitage peu élaborées avec l'existence de matières premières contraignantes, soit par leur médiocre aptitude à la taille, soit parce qu'elles se présentent selon des volumes peu favorables. Le débitage par percussion bipolaire sur enclume est, a priori, concerné par cette tendance compte tenu de son faible degré de complexité opératoire et des possibilités de contrôle limitées de la morphologie des produits. On le verrait ainsi se manifester lorsque le quartz, les quartzites ou des roches équivalentes sont les seules ressources lithiques et, plus encore, lorsqu'elles sont surtout disponibles sous la forme de galets, volontiers de petit module.
Pourtant, comme il a été souligné par les organisateurs de cette table-ronde, ce mode de production lithique est de plus en plus fréquemment reconnu dans des contextes chronologiques, culturels et géographiques extrêmement diversifiés où parfois ce sont des matières premières à texture fine qui ont été exploitées selon ce mode de débitage (Mourre et Jarry 2004).

Ounjougou est un vaste complexe de sites de plein air, en pays dogon (Mali) à quelques kilomètres à l'est de Bandiagara, dont l'occupation s'étend du Paléolithique ancien à l'époque actuelle. Son étude s'intègre dans le programme de recherche international pluridisciplinaire intitulé

(1) CNRS, UMR 7041, Maison de l'archéologie et de l'ethnologie, F-92023 Nanterre Cedex - sylvain.soriano@mae.u-paris10.fr

(2) Université de Fribourg, Avenue de l'Europe 20, CH-1700 Fribourg.

(3) Université de Genève, Département d'Anthropologie, CP 511, CH-1211 Genève 24. 
Paléoenvironnement et peuplement humain en Afrique de l'Ouest, coordonné par l'un des auteurs (E.H.) (Huysecom 2002 ; Huysecom et al. 2004a ; Huysecom et al. 2004b).

Pour le Paléolithique, les études archéologiques (placées sous la direction de S.S.), géomorphologiques et le programme de datations OSL permettent de restituer une séquence chronostratigraphique et chronoculturelle s'étendant sur plus de 100000 ans, de la fin du Pléistocène moyen au Pléistocène supérieur (Robert et al. 2003 ; Rasse et al. 2004 ; Soriano et Rasse 2005). Au sein de cette séquence, plus de quinze niveaux ou couches archéologiques ont été individualisés, dont les industries lithiques se rapportent au Paléolithique moyen / Middle Stone Age. Pour la plupart des occupations, les vestiges, strictement lithiques, sont conservés en position primaire ou peu dérivée dans des sédiments fins d'origine éolienne. Dans certains cas, on peut aussi les retrouver en position secondaire dans des dépôts fluviatiles.

L'utilisation de la percussion bipolaire sur enclume est avérée dans plusieurs niveaux archéologiques du Paléolithique moyen de la séquence d'Ounjougou dans un contexte géologique régional où les matières premières lithiques taillables sont peu variées, de qualité inégale et a priori contraignantes soit par leur accessibilité, soit par leur morphologie.

A partir de ce constat peuvent être posées deux questions simples, susceptibles d'éclairer le statut technique de la percussion bipolaire sur enclume pour les hommes du Paléolithique d'Ounjougou :

1) est-ce qu'il existe, dans la séquence d'Ounjougou, un lien net entre la mise en œuvre de la percussion bipolaire sur enclume et l'utilisation de matières premières "contraignantes» qui excluraient le recours à des modes de production plus élaborés ?

2) est-ce que les rôles technique et économique de la percussion bipolaire sur enclume sont similaires tout au long de la séquence d'Ounjougou?

Pour répondre à ces questions, il nous faudra au préalable présenter les critères sur lesquels se fondent notre diagnose de la percussion bipolaire sur enclume dans ces industries afin d'éviter des ambiguiités quant aux manifestations techniques que nous désignons ainsi mais aussi dresser un état des ressources en matières premières lithiques dans la région.

\section{PERCUSSION BIPOLAIRE SUR ENCLUME : CRITÈRES DE DIAGNOSE DANS LE PALÉOLITHIQUE D'OUNJOUGOU}

Devant la diversité des manifestations attribuées à la percussion bipolaire sur enclume et des stigmates afférents, soulignée lors de la table ronde, il apparaît opportun de préciser les critères sur lesquels nous avons fondé notre diagnose au sein des industries lithiques d'Ounjougou. Tous les stigmates observés plaident en faveur de la mise en œuvre de la percussion bipolaire sur enclume selon une configuration où la matière première lithique à tailler, immobilisée manuellement, repose sur une enclume minérale. Elle est alors percutée en un point opposé au contact avec l'enclume par un percuteur minéral dur mobile effectuant un mouvement linéaire vertical (fig. 1).

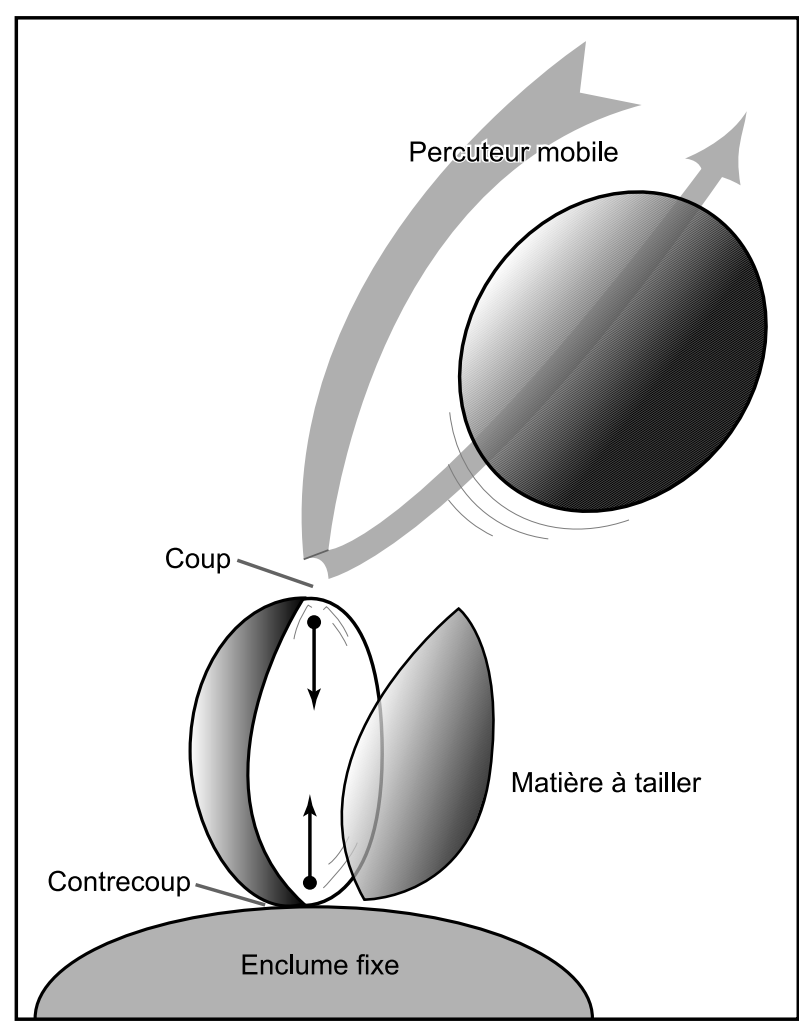

Figure 1 - Schéma de principe de la percussion bipolaire sur enclume telle que mise en œuvre dans les industries paléolithiques d'Ounjougou (Mali).

Figure 1 - Schema demonstrating the principle of the bipolar anvil technique as utilized in the Paleolithic industries of Ounjougou (Mali).

La présence d'éclats dont l'extraction a clairement été initiée en deux points à partir desquels l'onde de fracture a progressé de façon simultanée, selon un même axe mais avec des directions opposées, est ici l'élément déterminant de la diagnose de la percussion bipolaire sur enclume. L'un des points d'initiation de la fracture est lié à l'impact d'un percuteur minéral dur mobile tandis que l'autre est la manifestation du contrecoup au point de contact entre la matière taillée et l'enclume, elle aussi minérale. Il est le plus souvent impossible de distinguer spécifiquement les stigmates du coup de ceux du contrecoup. 

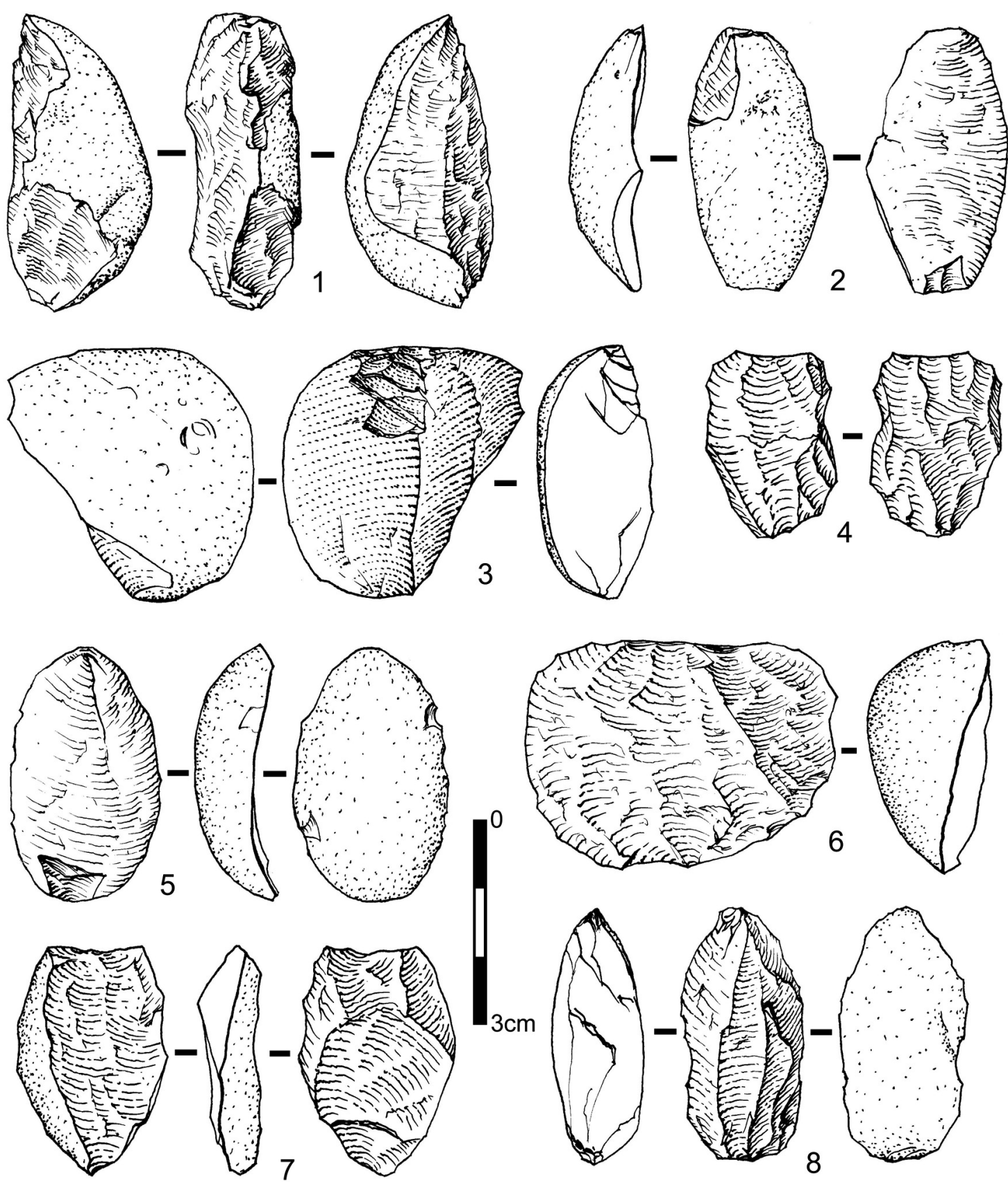

Figure 2 - Dandoli 1/3 (Ounjougou, pays dogon, Mali). Produits et sous-produits obtenus par percussion bipolaire sur enclume. 1,6 et 8 : nucléus, 2 à $5,7:$ éclats.

Figure 2 - Dandoli 1/3 (Ounjougou, Dogon Country, Mali). Products and by-products of the bipolar anvil technique. 1, 6 and 8: cores, 2-5, 7: flakes. 

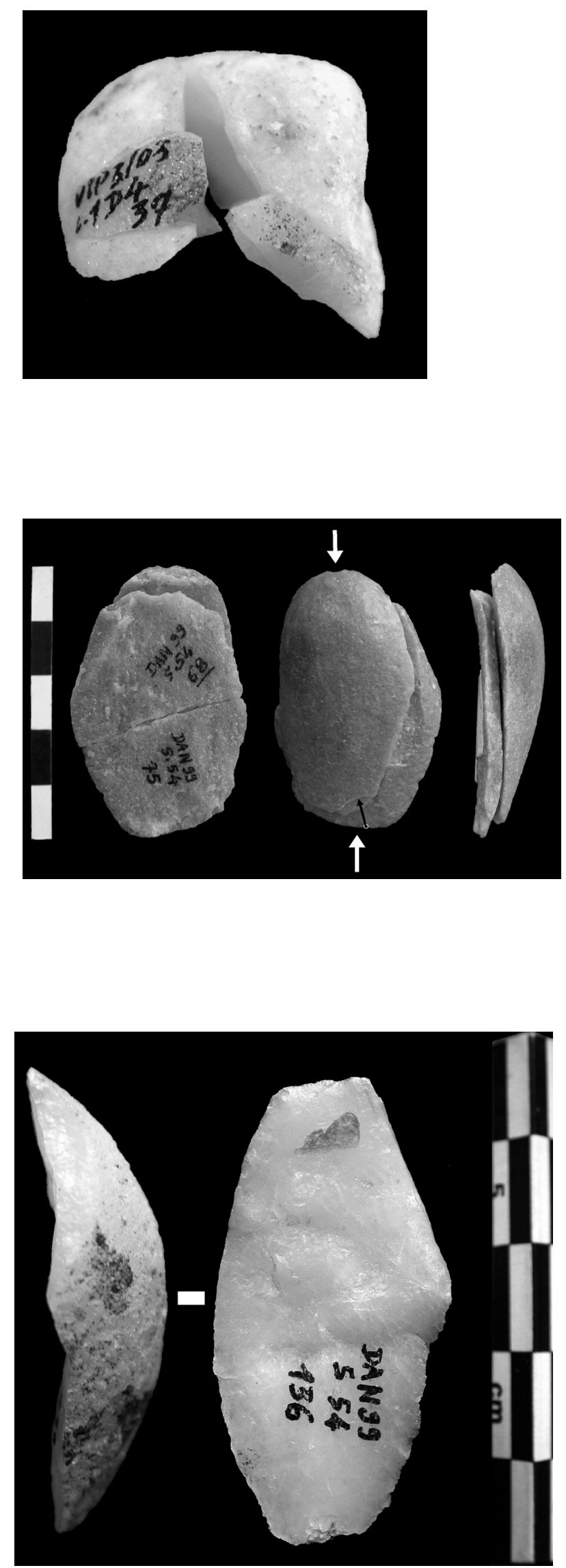

Figure 3 - Ravin de la Vipère C1 (Ounjougou, pays dogon, Mali). Remontage d'un galet taillé par percussion bipolaire sur enclume. Sur cette vue d'un des deux points d'initiation de la fracture (coup ou contrecoup ?) on observe nettement le développement radiaire de plusieurs plans de fracturation.

Figure 3 - Ravin de la Vipère C1 (Ounjougou, Dogon Country, Mali). Refitting of a cobble reduced by the bipolar anvil technique. In this view of one of the two initiation points of the fracture (striking blow or reflected force?), we clearly observe the radial development of multiple fracture planes.

Figure 4 - Dandoli 1/3 (Ounjougou, pays dogon, Mali). Remontage de trois fragments d'un éclat débité par percussion bipolaire sur enclume illustrant le phénomène de "dédoublement» dans l'épaisseur de l'éclat. Le négatif d'un esquillement sur la face supérieure (flèche noire), lié au coup ou au contrecoup car issu d'un point d'initiation de la fracture, recoupe à l'une de leurs extrémités les deux fragments superposés, montrant qu'ils résultent bien d'une unique percussion et de son contrecoup. A noter que la partie supérieure de l'éclat ne présente qu'un point d'initiation de la fracture.

Figure 4 - Dandoli 1/3 (Ounjougou, Dogon Country, Mali). Refitting of three flake fragments created by the bipolar anvil technique. The scar of a splinter on the upper face (black arrow), resulting from the striking blow or reflected force because it issues from an initiation point of the fracture, cuts across the two superimposed products to one of their ends, demonstrating that they were produced from a single blow and its reflected force. It should be noted that the upper part of the flake has only a single fracture initiation point.

Figure 5 - Dandoli 1/3 (Ounjougou, pays dogon, Mali). Profil et face inférieure d'un éclat obtenu par percussion bipolaire sur enclume. On note l'angle formé par l'axe de débitage et la face supérieure au niveau du point de percussion qui témoigne que le coup/contrecoup a été très fréquemment donné sur un plan faisant avec la surface débitée un angle nettement ouvert.

Figure 5 - Dandoli 1/3 (Ounjougou, Dogon Country, Mali). Profile and ventral surface of a flake obtained by the bipolar anvil technique. The angle formed by the fracture axis and the upper face at the striking point indicate that the blow/reflected force was quite frequently struck on a surface having a clearly obtuse angle with the surface being reduced. 

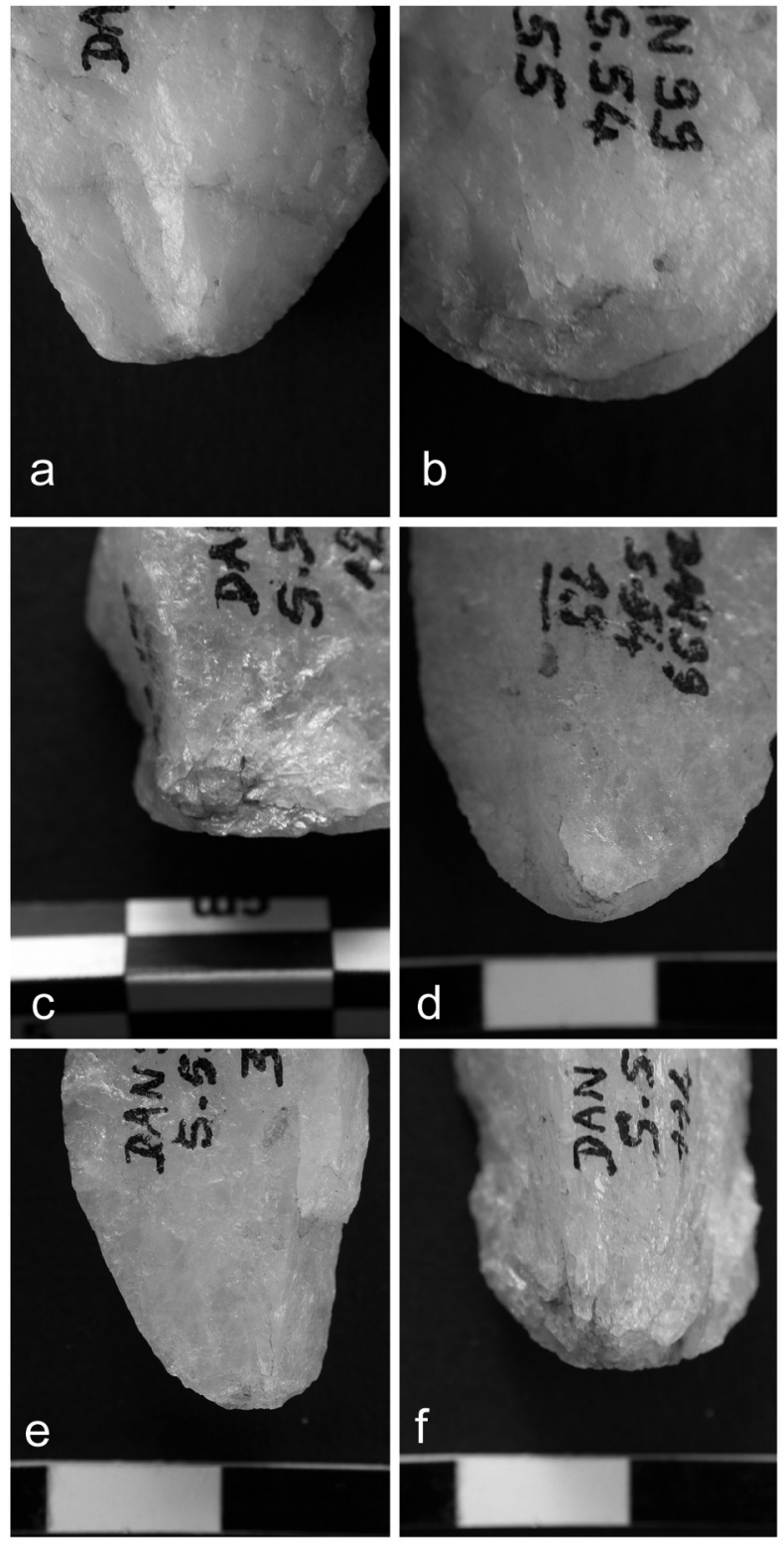

La face d'éclatement (face inférieure) des produits, sans bulbe ou avec un bulbe très discret, présente en général peu de relief. Ce fait est à mettre en rapport avec le mode de fracturation qui n'est pas conchoïdal ${ }^{4}$. II est ainsi parfois impossible de distinguer positif et négatif, éclat et nucléus ${ }^{5}$. Le profil longitudinal de la face d'éclatement peut être rectiligne, convexe ou concave. Des ondulations, voire des rides fines et serrées sur les matériaux les plus fins, sont parfois visibles sur cette face. La face d'éclate-
Figure 6 - Dandoli 1/3 (Ounjougou, pays dogon, Mali). Stigmates particuliers au point d'initiation de la fracture (coup ou contrecoup). a - bulbe peu proéminent, $b$ esquillement important, $c$ - écrasement, $d$ - petit esquillement, e - petit plan de fracture orthogonal, $f$ - écrasement et petites fissures radiaires.

Figure 6 - Dandoli 1/3 (Ounjougou, Dogon Country, Mali). Characteristic traits at the fracture initiation point (blow or reflected force). $a$ - shallow bulb, $b$ - major splintering, $c$ crushing, $d$ - minor splintering, e-small orthogonal facture plane, $f$-crushing and small radial fissures.

ment peut être composée de plusieurs pans sécants entre eux qui peuvent donner une section polygonale au produit / sous-produit (fig. $2, n^{\circ} 8$ ). Ces pans peuvent être nettement distincts dès les points d'initiation de la fracture et le rester tout au long de celle-ci ou, plus rarement, s'infléchir et se confondre à mi-course (fig. $2, n^{\circ} 5$ ). Leur existence relève peut-être du même moteur que pour les accidents de type Siret à plans multiples décrits sur le quartz par V. Mourre (Mourre 1996). Un des remontages que nous avons réalisé sur le matériel archéologique montre ainsi comment, à partir d'un unique coup/contrecoup donné dans l'axe longitudinal d'un galet de quartz ovoïde, plusieurs plans de fracturation radiaires se sont développés (fig. 3). Le développement d'un plan de fracturation secondaire parallèle à la face d'éclatement principal est aussi attesté. Ce dédoublement dans l'épaisseur de l'éclat peut n'affecter l'une des extrémités que sur quelques millimètres ou s'étendre jusqu'à l'extrémité opposée, ainsi que l'illustre un remontage (fig. 4).

Les produits indubitablement obtenus par percussion bipolaire sur enclume dans les industries d'Ounjougou n'ont, à quelques exceptions près, pas de talon, ou alors ce dernier se réduit à un point ou un fil à l'intersection de la face d'éclatement et de la face supérieure. Par ailleurs, si l'absence de talon ne permet pas de mesurer l'angle de chasse réel, tout indique, en particulier lorsque la face supérieure présente les convexités corticales du galet d'origine, que le coup/contrecoup a été très fréquemment donné sur une surface faisant avec la surface débitée un angle nettement ouvert, a priori peu favorable pour une percussion classique (fig. $2, n^{\circ} 2,3$ et 5 et fig. 5). Cela reste tout à fait cohérent avec le mode de fracturation non

(4) A notre connaissance, il n'existe pas d'antonyme à «conchoïdal» pour désigner ce mode de fracturation. On parle parfois de fracturation en «split» (Pelegrin, communication orale).

(5) Nous avons considéré comme «nucléus» les pièces présentant plusieurs faces d'éclatement auxquelles pouvaient être clairement associés au moins deux évènements de coup/contrecoup, de direction identique ou différente. C'est une définition a minima car un unique coup/contrecoup peut diviser le volume à tailler en plusieurs pièces de section polygonale assez identiques entre elles sans que l'on puisse réellement distinguer un nucléus et des éclats. 
conchoïdal que nous avons déjà évoqué. II faut toutefois noter que dans les industries d'Ounjougou la présence de produits présentant de tels caractères en lieu et place du talon n'est pas exclusivement liée à la pratique de la percussion bipolaire. II semble en effet que les stigmates d'une fracturation non conchoïdale, qui se serait développée à partir d'un unique point d'initiation, soient fréquemment observés lors de l'entame des petits galets de quartz localement disponibles, en particulier ceux dont la texture est microcristalline. Enfin, on peut penser que l'absence de talon aux deux pôles d'initiation de la fracture soit aussi liée à la localisation de l'impact du percuteur mobile sur la matière à tailler, vraisemblablement sur, ou très proche, de l'arête créée par l'intersection de la surface débitée avec la surface de plan de frappe ${ }^{6}$, ainsi qu'à la position de la matière à tailler sur l'enclume qui détermine la localisation du contrecoup.

Aux points d'initiation de la fracture, on observe fréquemment un écrasement de la matière, surtout lorsque le quartz a une structure grenue, des écaillures étagées qui peuvent affecter la face supérieure comme la face d'éclatement et des fissures radiaires d'ampleur variable. Parfois, on observe pour l'un des deux points d'initiation de la fracture un stigmate équivalent au fort esquillement bulbaire décrit sur les produits laminaires débités au percuteur de pierre tendre (Pelegrin 2000) (fig. 6).

Si les stigmates que nous avons observés sur les industries d'Ounjougou et décrits ici sont très caractéristiques de la percussion bipolaire sur enclume, mise en œuvre selon le procédé évoqué, ils ne sauraient être considérés sans distinction comme représentatifs de l'ensemble des manifestations de ce mode de taille. Ils en constituent plutôt une des multiples expressions potentielles.

\section{LES RESSOURCES EN MATIÈRES PREMIÈRES LITHIQUES SUR LE PLATEAU DOGON}

Bien que les ressources en matières premières lithiques aptes à la taille soient très abondantes sur le plateau de Bandiagara, elles sont assez peu diversifiées. La géologie du plateau, très monotone, explique cela. En effet, il s'agit d'un vaste entablement de grès fluvio-deltaïque ordovicien s'étendant sur plus de cent kilomètres, où des bancs gréseux décimétriques se succèdent régulièrement. Cette rythmicité des bancs gréseux est localement interrompue par des passées gréso-conglomératiques. La minéralogie et la granulométrie de ces grès, où le quartz dominant côtoie une fraction assez variable de feldspaths très souvent altérés (kaolinisés), les rend impropres à la taille. Localement cependant, la granulométrie bien plus fine et la rareté des feldspaths dans ces fractions fines, alliées à une diagenèse plus poussée, a engendré des bancs de grès plus tenaces et mieux cimentés dont la structure est parfois quartzitique. C'est un des matériaux qui a été taillé par les Paléolithiques. Les autres matières premières qui ont été taillés viennent des passées gréso-conglomératiques. II s'agit majoritairement de galets de quartz mais aussi des galets de quartzite (moins de $5 \%$ ) et, de façon anecdotique, des galets d'autres matériaux (silex, jaspe et autres roches siliceuses cryptocristallines principalement). Les galets de quartz, comme les sables qui les emballent, sont les résidus de l'érosion d'un très vaste socle primaire. Ils sont de ce fait de nature pétrographique très diversifiée et par conséquent de qualité très variable.

A plusieurs dizaines de kilomètres au nord-est du site, dans la région de Tassembé, existent des roches vertes de faciès paléomagmatique mais que l'on retrouve utilisées uniquement dans les industries du Néolithique sous la forme de lames de hache ou d'herminette polies.

Pour les ressources en matières taillables, on a donc globalement une opposition entre quartz et grès en termes de distribution spatiale, de disponibilité et de module. Les galets de quartz, abondants, sont omniprésents dans le paysage, aussi bien dans les dépôts fluviatiles que dans les altérites sur les affleurements du substrat gréseux. Leur qualité est variable, de très bonne à médiocre, en fonction de la texture du matériau, qui oscille entre microcristalline et grossièrement grenue. Les tailleurs néolithiques ont façonné par pression des armatures bifaciales dans les variétés de quartz les plus homogènes ce qui en souligne la qualité. On peut supposer que le transport fluviatile subi par ces matériaux dans leur milieu de dépôt à l'Ordovicien a contribué à éliminer les blocs diaclasés. Le module des galets de quartz oscille généralement entre 4 et $7 \mathrm{~cm}$ et n'atteint qu'exceptionnellement 8 à $10 \mathrm{~cm}$. Le grès apparaît à l'opposé en gîtes ponctuels dispersés sur le territoire et se présente sous la forme de bancs épais. II permet donc la fabrication de pièces massives à partir de fragments ou de gros éclats. Sa qualité est parfois excellente, permettant alors le façonnage de pièces bifaciales foliacées.

Hormis quelques cas dans les niveaux archéologiques les plus anciens, le grès quartzite a toujours été collecté sur les gîtes primaires. A l'opposé, pour les matériaux issus des passées gréso-conglomératiques, on note qu'une fraction importante des galets a été collectée sur des gîtes secondaires, au sein des dépôts fluviatiles de cours d'eau actifs au moment des occupations paléolithiques.

Les matériaux siliceux les plus fins (silex, jaspe) sont très rares et les Paléolithiques ne les ont pas spécifiquement recherchés avant la fin du Pléistocène supérieur. Une collecte ciblée sur ces matières premières ne semble apparaître dans la région qu'avec la confection d'armatures géométriques microlithiques.

\section{DE LA CONTRAINTE DES MATIÈRES PREMIÈRES...}

À une exception presque anecdotique puisqu'il s'agit d'un polyèdre fendu (fig. 7) la percussion bipolaire sur enclume a été exclusivement mise en œuvre pour traiter le quartz et les autres matériaux accessibles sous forme de petits galets, comme le quartzite. Le quartz est présent dans presque tous les niveaux archéologiques mais sa fréquence est très variable: de quelques pièces éparses en marge de débitage en grès (Vipère C2), à l'intégralité de l'industrie (Vipère C1, Orosobo 2 ou Kokolo 2 ; Soriano 2003).

(6) Cette configuration est rencontrée dès qu'a été extrait un premier enlèvement, cortical dans le cas du débitage de galets de quartz. 

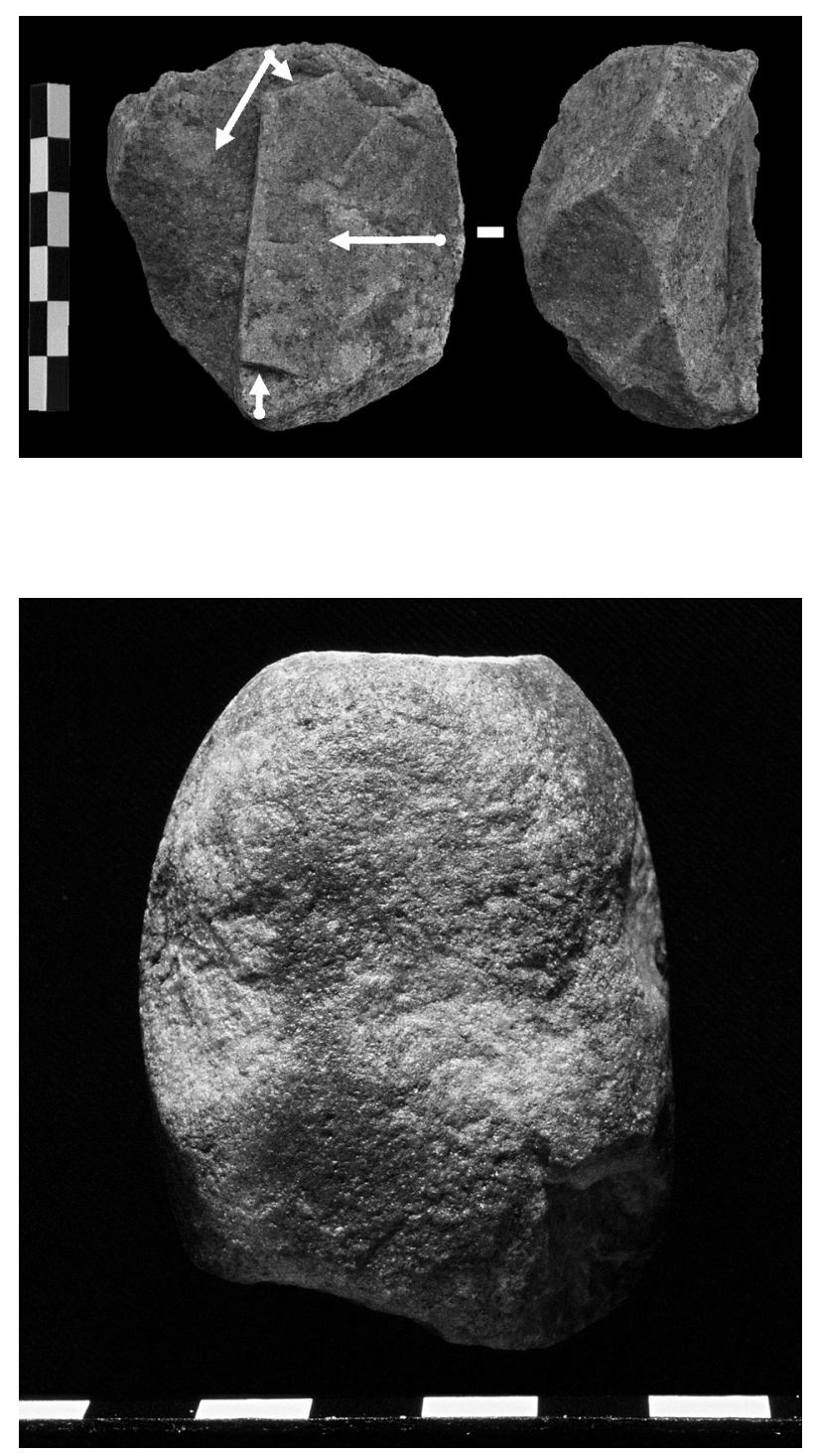

Surtout, malgré une matière première a priori contraignante, la percussion bipolaire sur enclume n'est pas incontournable. En témoignent les tailleurs d'Orosobo 2 qui ont pratiqué le débitage Levallois, à l'exclusion de toute autre méthode de débitage, sur les mêmes galets de quartz que leurs prédécesseurs.

\section{DU RÔLE DE LA PERCUSSION BIPOLAIRE SUR ENCLUME...}

Nous nous limiterons ici à développer deux exemples, Dandoli $1 / 3$ et Ravin de la Vipère $C 1$ où le statut technique et économique de la percussion bipolaire sur enclume apparaît très différent.

\section{Dandoli 1/3}

Le niveau archéologique identifié à Dandoli compte parmi les dernières occupations paléolithiques du plateau avant
Figure 7 - Confluence (Ounjougou, pays dogon, Mali). Exemple anecdotique de l'emploi de la percussion bipolaire sur enclume pour un matériau autre que les galets de quartz/quartzite. Polyèdre en grès fracturé par percussion bipolaire sur enclume. On note la succession de deux percussions d'axes orthogonaux.

Figure 7 - Confluence (Ounjougou, Dogon Country, Mali). Anecdotal example of the use of the bipolar anvil technique for raw material other than quartz/quartzite cobbles.

Sandstone polyhedron fractured by bipolar anvil percussion. Note the succession of two blows from orthogonal axes.

Figure 8 - Dandoli 1/3 (Ounjougou, pays dogon, Mali). Galet de siltite interprété comme ayant fonctionné en tant que enclume dans le débitage bipolaire qui caractérise cette industrie. II présente sur chacune de ses faces de multiples traces d'écrasement jointives qui forment de petites dépressions en forme de cupule (Cliché $n^{\circ} 99 D 0210$, service photographique CNRS UMS 844, MAE, Nanterre).

Figure 8 - Dandoli 1/3 (Ounjougou, Dogon Country, Mali). Siltite cobble interpreted as having been used as an anvil for the bipolar reduction characterizing this industry. It presents on each one of its faces, multiple jointed traces of crushing which form small depressions. (Photo $n^{\circ}$ 99D0210, photography service of CNRS UMS 844, MAE, Nanterre).

le dernier grand aride ogolien. II est positionné en stratigraphie au sein d'une séquence de silts sableux d'origine éolienne probablement redistribués dans une dynamique colluviale. Les datations OSL préliminaires de l'ensemble de la séquence sédimentaire situent l'occupation autour de 26000 / 27000 ans (Robert et al. 2003).

Le quartz domine très nettement l'assemblage bien que quelques produits en grès soient néanmoins présents. Parmi les produits en quartz (ou quartzite), ceux qui présentent des stigmates de taille indubitablement associés à la mise en œuvre de la percussion bipolaire sur enclume sont largement majoritaires (fig. 2). De façon originale à Ounjougou, cette technique devient l'élément structurant d'un schéma de débitage systématique ayant donné la plupart des supports débités, même si quelques nucléus n'ont manifestement pas été exploités ainsi. On retrouve d'ailleurs deux pièces lithiques qui ont vraisemblablement joué le rôle d'enclume, l'une entière (fig. 8), l'autre fragmentaire, comme le suggèrent les stigmates d'écrasement 
qu'elles portent et qui se concentrent jusqu'à former de petites dépressions en forme de cupule. L'essentiel des supports produits est resté brut. Seuls deux racloirs ont visiblement été confectionnés sur des éclats issus de la production bipolaire sur enclume.

\section{Ravin de la Vipère $\mathbf{C 1}$}

Les trois niveaux archéologiques du Ravin de la Vipère occupent la partie sommitale des dépôts de l'unité sédimentaire U4 et se placeraient autour de 50000 ans d'après les datations OSL préliminaires. Le niveau archéologique $\mathrm{C} 1$, qui nous intéresse ici, se marque par de petits amas de débitage, regroupant chacun entre une dizaine et une trentaine d'objets, dispersés sur une paléosurface au sein d'un semis épars de pièces isolées. La densité des esquilles et petits débris au sein des amas, la faible dispersion verticale ainsi que la fréquence des remontages montrent que ce niveau a été peu perturbé depuis son enfouissement.

L'industrie lithique est exclusivement réalisée à partir de petits galets de quartz collectés principalement en gîte primaire. Les pièces qui présentent des stigmates attribués à la percussion bipolaire sur enclume sont ici beaucoup plus rares que dans l'industrie de Dandoli. Elles sont même très minoritaires devant les produits issus d'un débitage de modalité discoïde qui a donné des éclats courts, assez larges et à talon épais, fréquemment corticaux. Comme cela a déjà été mentionné dans d'autres contextes, les tailleurs ont volontiers conservé la surface corticale des galets de quartz comme plan de frappe pour mener leur débitage discoïde (Jaubert et Mourre 1996).

Quelques remontages ont permis d'éclairer les relations entre les deux modes de taille (fig. 9). Certains galets de quartz ont manifestement été fractionnés par percussion bipolaire sur enclume et les fragments au volume adéquat, prélevés, ont été débités selon une modalité discoïde. Parallèlement, on observe aussi une production indépendante par percussion bipolaire sur enclume, mais qui ne semble avoir donné que quelques produits, ainsi que la présence de quelques pièces esquillées, lesquelles se différencient assez nettement du débitage.

Outre les pièces esquillées, l'outillage retouché est très rare. II s'agit uniquement de racloirs exclusivement réalisés par retouche inverse sur des éclats ou fragments corticaux (surface néo-corticale des galets de quartz), ce qui assure une certaine régularité au tranchant. Les produits spécifiquement obtenus par le débitage discoïde sont restés bruts.

\section{DU STATUT DE LA PERCUSSION BIPOLAIRE SUR ENCLUME À OUNJOUGOU}

Les deux exemples examinés suggèrent que le statut de la percussion bipolaire sur enclume est variable dans les industries du Paléolithique moyen d'Ounjougou. La percussion bipolaire sur enclume est rarement absente des industries d'Ounjougou (tabl. 1). Lorsque le grès a été seul utilisé (Oumounaama atelier "amas 1996») on comprend qu'elle soit absente, cette matière première se prêtant mal à la percussion bipolaire sur enclume. II est net que celle-ci a été mise en œuvre à Ounjougou par les

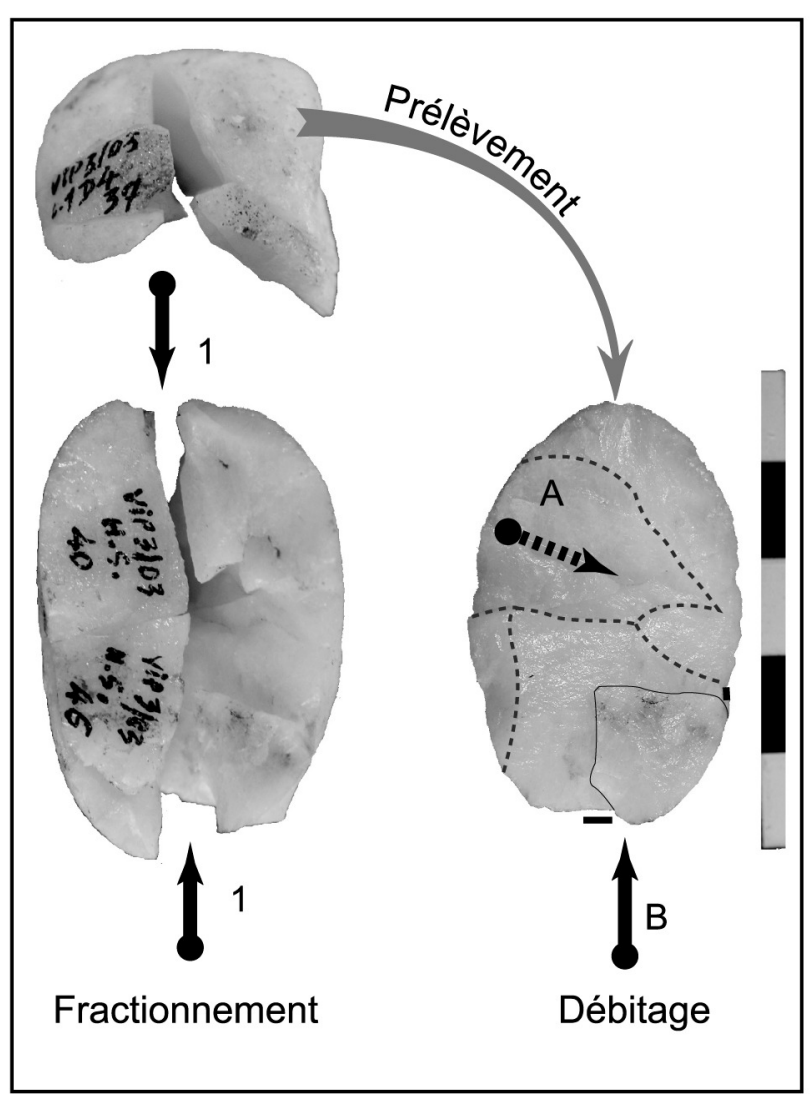

Figure 9 - Ravin de la Vipère C1 (Ounjougou, pays dogon, Mali). Un remontage éclaire les relations entre la percussion bipolaire sur enclume et le débitage de modalité discoïde. Un galet de quartz ovoïde a été fractionné par percussion bipolaire sur enclume (vue de gauche) puis le plus large fragment, prélevé, a été débité sur sa face de fracture selon une modalité discoïde (vue de droite). Des deux éclats extraits ( $A$ et $B)$, un fragment du second a été remonté.

Figure 9 - Ravin de la Vipère C1 (Ounjougou, Dogon Country, Mali). A refit sequence clarifies the relationship between the bipolar anvil technique and the discoid method. An ovoid quartz cobble was fractured by the bipolar anvil technique (left view), then the largest fragment obtained was reduced on its fracture face using the discoid method (right view). Of the two flakes produced ( $A$ and $B$ ), a fragment of the second could be refitted.

Paléolithiques presque systématiquement dès lors que le quartz, sous forme de galets, est utilisé qu'il soit à dessein ou par défaut la matière première dominante (tabl. 1). II existe cependant un cas (Orosobo 2) où le quartz est la matière première exclusive mais pour lequel la percussion bipolaire sur enclume n'a pas été utilisée. Cela démontre que la contrainte liée à la matière première ne peut être seule invoquée pour expliquer le recours à la percussion bipolaire sur enclume. 


\begin{tabular}{|c|c|c|c|c|}
\hline & \multirow{2}{*}{\multicolumn{2}{|c|}{$\mathbf{A}$}} & \\
\hline & & & $\mathbf{B}$ & $\mathbf{C}$ \\
\hline & \multicolumn{2}{|c|}{ MP } & \multirow{2}{*}{$\begin{array}{c}\text { Rôle de la } \\
\text { perc. } \\
\text { bip./encl. }\end{array}$} & \multirow{2}{*}{$\begin{array}{l}\text { Débit. avec } \\
\text { perc. bip/encl. }\end{array}$} \\
\hline & Quartz & Grès & & \\
\hline Dandoli 1/3 & ++ & + & $\mathrm{T}$ & ++ \\
\hline Vipère C1 & +++ & $\varnothing$ & $\mathrm{F}, \mathrm{T}$ & + \\
\hline Vipère C2 & + & ++ & $\mathrm{T}$ & $?$ \\
\hline Orosobo & + & ++ & $\mathrm{T}$ & $?$ \\
\hline Orosobo 2 & +++ & $\varnothing$ & & $\varnothing$ \\
\hline Oumounaama 2 niv. sup. & +++ & $\varnothing$ & & $\varnothing$ \\
\hline Oumounaama 2 niv. inf. & ++ & + & $\mathrm{T}$ & + \\
\hline Draperies C5 & ++ & + & $\mathrm{T}$ & + \\
\hline Oumounaama ouest & ++ & + & $\mathrm{T}$ & + \\
\hline Ravin sud 2 & +++ & $\varnothing$ & $\mathrm{T}$ & + \\
\hline Oumounaama atelier "amas" 1996 & $\varnothing$ & +++ & & $\varnothing$ \\
\hline Kokolo 2 & +++ & $\varnothing$ & $\mathrm{T}$ & + \\
\hline \multicolumn{5}{|l|}{ B } \\
\hline \multicolumn{5}{|c|}{$\begin{array}{l}\mathbf{T} \text { : la percussion bipolaire sur enclume est la technique de taille exclusive dans un } \\
\text { schéma de débitage simple }\end{array}$} \\
\hline \multicolumn{5}{|c|}{$\begin{array}{l}\text { F : la percussion bipolaire sur enclume est une technique utilisée dans la phase } \\
\text { d'initialisation d'un schéma de débitage élaboré }\end{array}$} \\
\hline
\end{tabular}

\begin{tabular}{|c|}
\multicolumn{1}{c|}{ A et $\mathbf{C}$} \\
\hline+++ Exclusif \\
++ Majoritaire \\
+ Minoritaire \\
$?$ Présence possible \\
$\varnothing$ Absence \\
\hline
\end{tabular}

Tableau 1 - La percussion bipolaire sur enclume dans les industries paléolithiques d'Ounjougou. Synthèse.

A : proportion de quartz (galets de quartz, quartzite et équivalents) et de grès dans l'industrie, $B$ : rôle de la percussion bipolaire sur enclume, $C$ : fréquence du schéma de débitage sur quartz utilisant la percussion bipolaire sur enclume comme technique exclusive par rapport aux autres schémas de débitage.

Table 1 - The bipolar anvil technique in the Ounjougou Paleolithic industries. Summary. A: proportions of quartz (cobbles of quartz, quartzite and equivalents) and sandstone in the industry, B: function of bipolar anvil technique, C: frequency of the reduction sequence on quartz with the exclusive use of bipolar anvil technique in relation to other reduction methods.

Le statut technique de la percussion bipolaire est aussi variable puisqu'elle peut être soit mise en œuvre exclusivement tout au long d'un schéma de débitage assez simple, ce qui est le cas le plus fréquent, soit être une modalité d'initialisation particulière à l'amont de schémas de débitage plus élaborés, tel le discoïde, comme le montre l'exemple du Ravin de la Vipère $\mathrm{C} 1$.

Par ailleurs, sa contribution économique est assez variable. Même si dans l'état actuel des recherches nous ne pouvons comparer la fréquence des produits obtenus par percussion bipolaire sur enclume dans les assemblages des différents sites puisque plusieurs études sont encore en cours, des différences apparaissent tout de même lorsqu'on détermine simplement si la percussion bipolaire sur enclume est minoritaire, majoritaire ou exclusive dans la production lithique débitée (tabl. 1). Dans un seul cas (Dandoli 1/3), la plupart des supports débités l'ont été par percussion bipolaire sur enclume. Dans les autres cas où elle a été mise en œuvre comme technique exclusive dans un schéma de débitage, elle accompagne une production plus élaborée dans ses modalités opératoires, sur quartz ou sur grès.

\section{CONCLUSION}

Nous constatons que malgré des ressources en matière première limitées dans leur nature, bancs de grès ou petits galets de quartz, les réponses techniques apportées par les Paléolithiques dans la production lithique apparaissent variées. La percussion bipolaire sur enclume apparaît ici comme une réponse parmi un ensemble de possibilités. Sa mise en œuvre prend alors une valeur de choix manifeste de la part des Paléolithiques d'Ounjougou puisqu'elle n'est pas techniquement absolument nécessaire. Si nous avons répondu à la question du statut économique et technique de la percussion bipolaire sur enclume dans les industries paléolithiques d'Ounjougou, le sens des différences n'est pas pour autant explicité. Pourquoi certains groupes ont utilisé la percussion bipolaire sur enclume pour traiter les petits galets de quartz d'Ounjougou et d'autres non ? Ce mode de taille, peu élaboré, était-il accepté et valorisé dans le bagage technique de tous les tailleurs paléolithiques ? Ces questions restent ouvertes. Pour terminer, on peut penser que le degré de contrainte imposé par les matières premières d'Ounjougou est suffi- 
samment élevé pour expliquer la présence marquée de la percussion bipolaire sur enclume sans toutefois faire de ce mode de taille l'unique solution techniquement possible.

Pour le Paléolithique moyen en Afrique de l'Ouest, en dehors d'Ounjougou, nous n'avons pas encore identifié dans la littérature d'autres industries où la percussion bipolaire sur enclume ait été reconnue. Les contributions rassemblées ici démontrent qu'elle est présente dans des contextes très diversifiés, comme par exemple dans plusieurs assemblages du Paléolithique moyen du Latium méridional en Italie (Bietti et al., ce volume).

\section{Remerciements}

La mission archéologique française d'Ounjougou, placée sous la direction de Sylvain Soriano, est soutenue financièrement par le Ministère des Affaires étrangères. Elle s'insère dans un programme de recherche international intitulé Paléoenvironnement et peuplement humain en Afrique de l'Ouest coordonné par Eric Huysecom et financé par la Fondation Suisse Liechtenstein pour l'Archéologie (FSLA), le Fond National de la Recherche Scientifique (FNRS) et l'État de Genève. Sylvain Soriano a reçu en 2002 pour ses recherches le soutien de la fondation Fyssen par l'attribution d'une bourse post-doctorale. Cette recherche a par ailleurs bénéficié en 2004 et 2005 du Programme d'Action Intégré (PAl) franco-suisse Germaine de Staël.

Nous saluons vivement nos partenaires maliens pour leur collaboration : Kléna Sanogo (Institut des Sciences Humaines), Doulaye Konaté et Daouda Keita (Univ. Bamako, Faculté des Lettres, Arts et Sciences Humaines), Lassana Cissé (Mission culturelle de Bandiagara) ainsi que les villageois de Dimbal, Gologou, Kokolo et Dandoli qui ont participé aux travaux de terrain.

Nous remercions enfin les deux relecteurs dont les suggestions ont contribué à améliorer cet article.

\section{BIBLIOGRAPHIE}

HUYSECOM E. 2002 - Palaeoenvironnement and human population in West Africa: an international research project in Mali. Antiquity, 76, p. 335-336.

HUYSECOM E., BALLOUCHE A., CISSÉ L., GALLAY A., KONATÉ D., MAYOR A., NEUMANN K., OZAINNE S., PERRET S., RASSE M., ROBERT A., ROBION C., SANOGO K., SERNEELS V., SORIANO S., STOKES S. 2004a Paléoenvironnement et peuplement humain en Afrique de l'ouest : rapport de la sixième campagne de recherche à Ounjougou (Mali). SLSA - Jahresbericht, 2003. Zurich, Vaduz : Fondation Suisse-Liechtenstein pour les Recherches Archéologiques à l'Etranger, p. 27-68.
HUYSECOM E., MAYOR A., OZAINNE S., RASSE M., SCHAER K., SORIANO S. 2004b - Ounjougou : plus de 100000 ans d'histoire en pays dogon (Mali). Archéologie suisse, 27, 2004, 3, p. 2-13.

JAUBERT J., MOURRE V. 1996 - Coudoulous, Le Rescoundudou, Mauran : Diversité des matières premières et variabilité des schémas de production d'éclats. In : A. Bietti et S. Grimaldi, eds., Reduction processes for the european mousterian. p. 313-341, (Quaternaria Nova, VI).

MOURRE V. 1996 - Les industries en quartz au Paléolithique. Terminologie, méthodologie et technologie. Paléo, 8, p. 205-224.

MOURRE V., JARRY M. 2004 - État du domaine et bibliographie de référence. In : V. Mourre et $M$. Jarry, éds., "Entre le marteau et l'enclume... ». Table ronde sur la percussion directe au percuteur dur et la diversité de ses modalités d'application. Résumés des communications, Toulouse, p. 2-10.

PELEGRIN J. 2000 - Les techniques de débitage laminaire au Tardiglaciaire : critères de diagnose et quelques réflexions. In : B. Valentin, P. Bodu et M. Christensen, éds., L'Europe centrale et septentrionale au Tardiglaciaire. Actes de la table-ronde internationale de Nemours, 14-16 mai 1997. Nemours : Ed. APRAIF, p. 73-86, (Mémoires du Musée de Préhistoire d'lle de France, 7).

RASSE M., SORIANO S., TRIBOLO C., STOKES S., HUYSECOM E. 2004 - La séquence pléistocène supérieur d'Ounjougou (Pays Dogon, Mali, Afrique de l'Ouest) : évolution géomorphologique, enregistrements sédimentaires et changements culturels. Quaternaire, 15, 4, p. 329-341.

ROBERT A., SORIANO S., RASSE M., STOKES S., HUYSECOM E. 2003 - First chrono-cultural reference framework for the West African Palaeolithic: new data from Ounjougou (Dogon Country, Mali). Journal of African Archaeology, vol. 1, (2), p. 151-169.

SORIANO S. 2003 - Quand archaïque n'est pas ancien ! Étude de cas dans le Paléolithique du Pays dogon (Ounjougou, Mali). Annales de la Fondation Fyssen, 18, p. 79-92.

SORIANO S., RASSE M. 2005 - Avant les Dogon : le site d'Ounjougou (Mali). In : Archéologies. Vingt ans de recherches françaises dans le monde. Paris : Maisonneuve et Larose, ADPF, ERC, p. 306-308. 